\title{
Analysis of the AC-DC harmonic interaction between the power network and uncontrolled 6 pulse rectifiers working in the discontinuous conduction mode using Newton's method
}

\author{
J.M. Cano, G.A. Orcajo, C.H. Rojas, M.G. Melero, M.F. Cabanas \\ Electrical Engineering Department \\ University of Oviedo \\ Campus de Viesques s/n - 33204 Gijón, Asturias (Spain) \\ phone:+34 985 182625, fax:+34 985 182453, e-mail: jmcano@correo.uniovi.es
}

\begin{abstract}
This paper summarises a study completely developed in [1] for the analysis of AC-DC harmonic interaction between the power network and non-controlled three-phase rectifiers operating in discontinuous mode. The presented method is an adaptation for the case of uncontrolled three-phase rectifiers of the procedure designed by Smith and Arrillaga [2] for the analysis of three-phase controlled rectifiers. The new algorithm has been developed in the frequency domain, and therefore introduces some important advantages for overall power system analysis. The influence of factors such as unbalance or harmonic distortion in the power supply voltage, harmonic distortion in the DC side voltage and unbalance in the $\mathrm{AC}$ side filtering reactances can easily be analysed. Moreover, a very flexible modelling of the circuits in both the DC and AC side can be achieved with a minimum effort.
\end{abstract}

\section{Key words}

Harmonics, rectifiers, discontinuous conduction mode, Newton's method.

\section{Introduction}

In the design and analysis of electric power systems it is extremely important to find out the effects of the interaction between the linear network and the non-linear devices. To achieve this, it is necessary to obtain very detailed models of such non-linear devices in order to integrate them into the network for an iterative mathematical resolution process.

For the case of six pulse rectifiers, several techniques exist to calculate the solution for the steady state in the frequency domain. The first of these techniques is the "fixed point iteration method". This algorithm uses the supply voltage of the rectifier as a starting point to predict the current it injects into the power system. These new currents, when imposed to the AC circuit generate a new voltage system that is then used as the starting point for a new iteration.

The main drawback of this fixed-point technique is its limited convergence capacity. In fact, when the power system is weak in the coupling point and/or the filtering reactances present a low value, the convergence of the algorithm cannot be assured [3]. Several solutions have been proposed to overcome this problem [2,pp.242]. However, all of them considerably increase the complexity of the calculation process. For this reason, present research tendencies focus on the substitution of the fixed-point algorithm for alternative calculation methods rather than finding solutions to its inherent convergence limitations.

The Equivalent Norton Method [2] models the circuit's non-linear devices during the iterative process as current sources parallel connected to an admittance. This admittance must be calculated to represent the device linearization around its operating point. In this way, the response of the algorithm during the iterative procedure is much faster and its convergence probability is higher.

In this method, an admittance matrix must be calculated to represent the dependency that exists at the operating point, between the supply current and every single harmonic voltage of the non-linear device. Although this method can be successfully applied for the modeling and analysis of certain electrical devices such as the study of saturation in power transformers, it is not suitable for the study of AC/DC converters since the influence of the DC side is neglected.

In 1989 E. V.Larsen [4] presented a method that sought to solve the interaction problem between the AC and DC sides of three-phase controlled rectifiers. This method, initially called HMAT (Harmonic Matrix Calculation) is now known as the " $A B C D$ parameters method". In this method, the linearization of the converter around its operation point is also applied. To do this, a set of new matricial equations is defined. These equations include the mathematical relationships between the converter supply voltage and currents, as well as the relationship of these variables with the DC voltage and current. The presence of control angle modulation, neglected in the Norton Equivalent Method, is also included in this model.

Although the matrixes that represent the increments in the system variables are obtained in a numerical fashion, this method neglects the effects of delayed commutation. For 
this reason, if this effect is to be included, the system of equations must be decoupled to be solved. In this case, the algorithm diverges if the rectifier supply voltages include even harmonics [5].

The Newton method, which will be described in the next section, solves the drawbacks related to the above calculation techniques. However, computation time for this algorithm is higher than for the ones referred to above.

\section{The Newton Method}

In 1996 Bruce C. Smith [6] proposed a solution exclusively based on the Newton method for the modelling of the three-phase controlled rectifier operating in continuous conduction mode. The main contribution of his study was the overall simultaneous treatment of both the rectifier's internal variables and their interaction with the AC and DC sides of the system. The theoretical principles of the Newton method are very simple, since it consist of the resolution of a non-linear equation system including all the electrical relationships between all the electrical variables of the rectifier. The overall application of this algorithm is presented in [2].

The new formulation needed for the application of the Newton method to non-controlled rectifiers operating in discontinuous mode has been developed in [1] by using the model presented in [7]. The AC side circuit is modelled as the three phase Thevenin's equivalent shown in Figure 1. This equivalent circuit can be used for the simplified representation of any linear power supply.

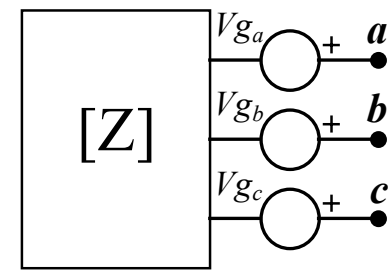

Fig. 1. Thevenin's equivalent of the AC side of the system

The DC circuit will also be represented by means of its Thevenin's equivalent. This circuit will be obtained for every supply frequency as the series combination of an impedance and an ideal voltage source (Fig. 2). Although such a circuit is linear, the application of additional iterative methods provides a solution even for non-linear equivalents.

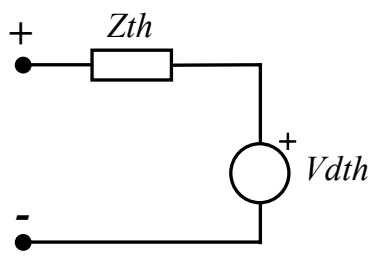

Fig. 2. Thevenin's equivalent of the DC side of the system

\section{A. Error Functions}

In this section, the complete set of equations that must be simultaneously solved in order to obtain the values of all the system variables will be described. These functions are all defined in the form $f(x)=0$ and are called error functions. The error functions can be classified into the following groups:

- Error functions for the starting of conduction $\left(F_{\theta i}\right)$

- Error functions for the end of conduction $\left(F_{\phi i}\right)$

- Error functions for the AC circuit $\left(F_{V_{k}^{\alpha}}\right)$

- Error functions for the ripple in the DC circuit $\left(F_{V d_{k}}\right)$

- Error functions for the DC circuit $\left(F_{V d_{0}}\right)$

- Error functions for the mean value of DC voltage $\left(F_{V d_{0}}\right)$

Table I shows the number of equations that must be solved for every type of error function. The index $\mathrm{N}$ represents the number of harmonics included in the solution.

TABLE I. - Number of equations to be solved

\begin{tabular}{|c|c|}
\hline $\begin{array}{c}\text { Group of Error } \\
\text { Function }\end{array}$ & Number \\
\hline$F_{\theta i}$ & 6 \\
\hline$F_{\phi i}$ & 6 \\
\hline$F_{V_{k}^{\alpha}}$ & $6 N$ \\
\hline$F_{V d_{k}}$ & $2 N$ \\
\hline$F_{V d_{0}}$ & 1 \\
\hline TOTAL & $\mathbf{8 N + 1 3}$ \\
\hline
\end{tabular}

\section{B. Mathematical description of the method}

The junction of all the error functions will produce a system of equations whose variables will be the $\mathrm{AC}$ supply voltages, the DC voltage and the starting and end of conduction times. The AC voltages are complex variables, for this reason its real and imaginary parts must be taken into account in the solution of the system. In this way, the total number of equations that must be solved is $8 \mathrm{~N}+13$, which corresponds to: $V_{k}^{\alpha}, V d_{k}, \theta_{i}, \phi_{i}, V d_{0}$.

The application of the Newton method to the above equation system produces matricial equation 1 .

$$
[S]^{n+1}=[S]^{n}+\left([J]^{n}\right)^{-1}[F]^{n}
$$

In this equation [S] is a column vector formed by all the system variables, $[\mathrm{F}]$ is a column vector formed by all error functions, and $[\mathrm{J}]$ is the Jacobian Matrix of the equation system. The index $n$ represents the number of iterations. 


$$
\left[\begin{array}{l}
R\left\{V_{k}^{a}\right\} \\
I\left\{V_{k}^{a}\right\} \\
R\left\{V_{k}^{b}\right\} \\
I\left\{V_{k}^{b}\right\} \\
R\left\{V_{k}^{c}\right\} \\
I\left\{V_{k}^{c}\right\} \\
R\left\{V d_{k}\right\} \\
I\left\{V d_{k}\right\} \\
\phi_{i} \\
\theta_{i} \\
I\left\{V d_{0}\right\}
\end{array}\right]^{n+1}\left[\begin{array}{l}
R\left\{V_{k}^{a}\right\} \\
I\left\{V_{k}^{a}\right\} \\
R\left\{V_{k}^{b}\right\} \\
I\left\{V_{k}^{b}\right\} \\
R\left\{V_{k}^{c}\right\} \\
I\left\{V_{k}^{c}\right\} \\
R\left\{V d_{k}\right\} \\
I\left\{V d_{k}\right\} \\
\phi_{i} \\
\theta_{i} \\
I\left\{V d_{0}\right\}
\end{array}\right]^{n}+\left([J]^{n}\right]^{-1}\left[\begin{array}{l}
R\left\{F_{V_{k}^{a}}\right\} \\
I\left\{F_{V_{k}^{a}}\right\} \\
R\left\{F_{V_{k}^{b}}\right\} \\
I\left\{F_{V_{k}^{b}}\right\} \\
R\left\{F_{V_{k}}\right\} \\
I\left\{F_{V_{k}^{c}}\right\} \\
R\left\{F_{V_{d_{k}}}\right\} \\
I\left\{F_{V d_{k}}\right\} \\
F_{\phi_{i}} \\
F_{\theta_{i}} \\
I\left\{F_{V d_{0}}\right\}
\end{array}\right]^{n}
$$

Equation 2 represents the expansion of equation 1. In this new expression the ordering of the different variables inside the matrixes can be observed.

In the next figure a flux diagram summarizing the different steps that it is necessary to follow in order to implement the described algorithm is presented. The block named "initial estimation of all the variables" initiates the calculation using the following assumptions: the rectifier supply voltage is identical to the Thevenin's equivalent voltage and the voltage ripple in the DC side is nil. The value of the DC voltage is estimated as slightly lower than the maximum possible value. This estimation implies a good approximation to its real value especially in DC circuits with a capacitive filtering stage.

The times of starting and end of conduction will be exactly calculated from the rectifier supply voltage and the DC side voltage after solving by the Newton-Raphson method the error functions $F_{\theta_{i}}$ y $F_{\phi_{i}}$.

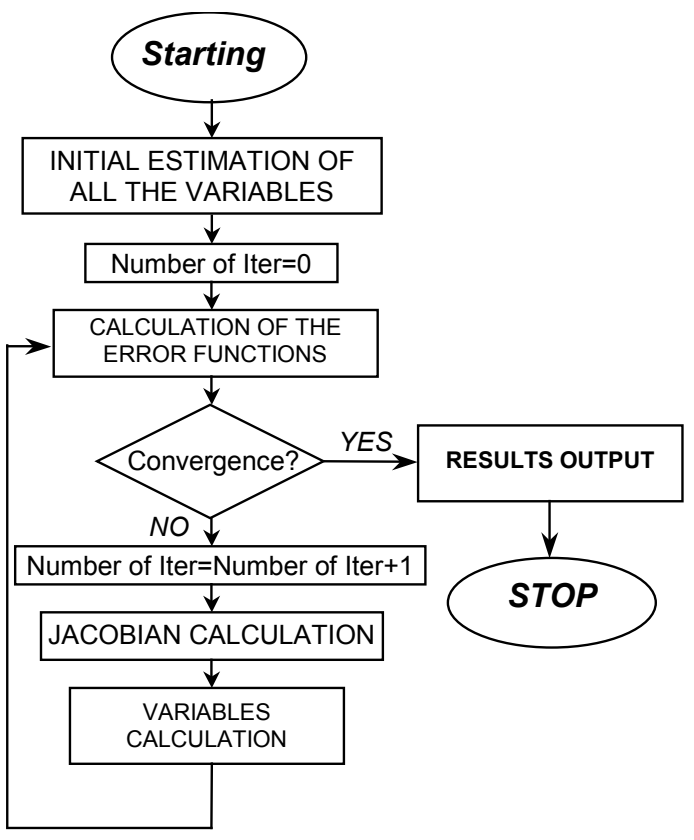

Fig. 3. Flux diagram for Newton's method application
The convergence of the iterative method will be reached when all the error functions produce output values close to 0 . In this way, it is possible to establish relative limits for every error function according to the following expression:

$$
\frac{\left|F_{X}\right|}{|X|}<\varepsilon
$$

Where $\varepsilon$ defines the degree of precision achieved in the solution. By means of this relative error parameter the same precision can be selected for all the harmonic components independently of their order and/or amplitude. Therefore, the exact estimation of all the harmonic components, even those that exhibit lower amplitudes, will be easily achieved. However, only the convergence of harmonic components whose amplitudes surpass a certain minimum value can be forced. If this restriction is not taken into account the iterative solution of the system would produce false harmonic components that are not really present in the system and this situation would give rise to numerical problems during the iteration process.

When it is not necessary to obtain the same exactitude in the calculation of all the harmonic components, other different approaches for the calculation of the solution are more suitable. For instance, establishing a limit for the sum of the absolute values of the deviation in every error function is a condition that can be very efficiently evaluated according to the following equation:

$$
\sum_{i=1}^{8 N+13}\left|F_{i}\right|<\varepsilon
$$

\section{Calculation of the Jacobian matrix of the system}

For the iterative solution of the equation system using the Newton method it is necessary to calculate the Jacobian matrix. This matrix, which is formed by the partial derivatives of every function with respect to every variable, can be calculated in two different ways. The first possible procedure consists of the numerical calculation of every partial derivative. In this case, the derivative of an error function $F$ with respect to the variable $x$ will be calculated by evaluating the error function in $x$ and $x+\Delta x$ according the equation (5):

$$
\frac{\partial F}{\partial x}=\lim _{\Delta x \rightarrow 0} \frac{F(x+\Delta x)-F(x)}{\Delta x} \approx \frac{F(x+\Delta x)-F(x)}{\Delta x}
$$

The application of this calculation procedure to every partial derivative will lead to the complete computation of the Jacobian matrix. However, this approach to the problem is not practical since the computation time required for the calculation of all the partial derivatives is extremely high. For this reason, a different non-numerical method must be applied.

The alternative to the numerical calculation of the partial derivatives is to obtain analytical expressions by means of the derivation of the error functions. Although this method initially involves a huge mathematical effort, it dramatically reduces computation time of the Jacobian matrix. In the present study the mathematical expressions of all the partial derivatives of the error functions have 
been calculated and can be checked in [1]. Moreover, all of them have been verified by using a numerical calculation method. Once the analytical expression of the partial derivative of every error function has been obtained its numerical value was compared with the numerical method defined by equation (5), and a perfect adjustment between both results was obtained. The exactitude of all the mathematical calculations was thus verified.

\section{Interpretation of the Jacobian matrix}

The Jacobian matrix obtained from the iterative solution of the equation system represents a linearisation of the represented electrical system around its operating point. Figure 4 shows the three-dimensional view of the Jacobian matrix obtained, including 13 harmonic components. In this figure the components of the matrix with values higher than 0.05 have been truncated in order to appreciate the influence of the less significant components.

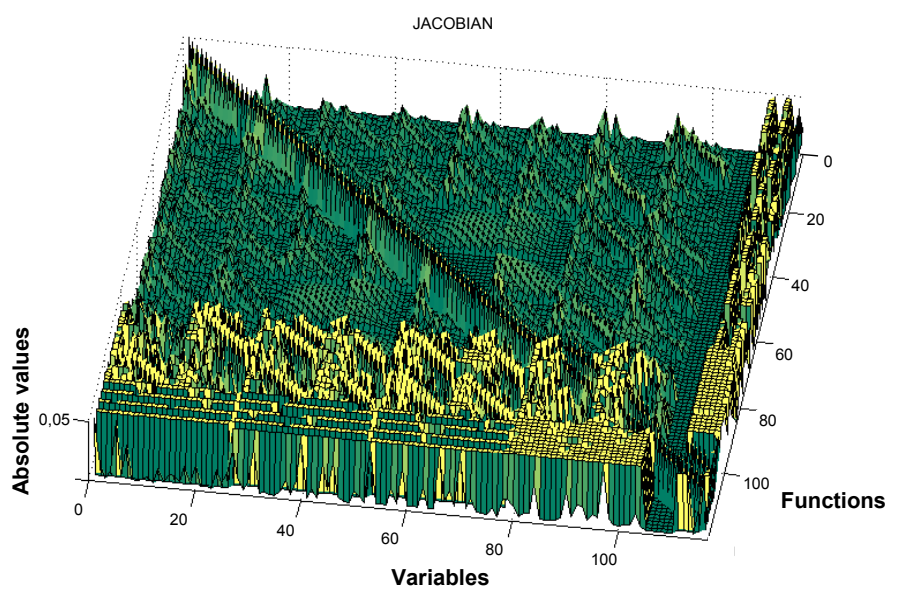

Fig. 4. Three-dimensional view of the Jacobian matrix

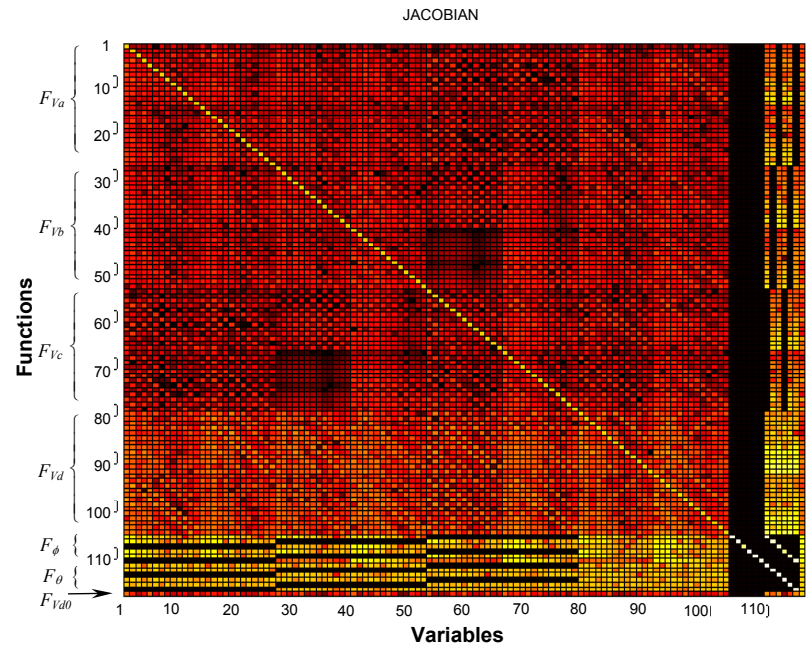

Fig. 5. Top view of the Jacobian matrix

A clearer evaluation of the relative importance of every component in the Jacobian matrix can be obtained by using the representation adopted in figure 5, where the top view of the Jacobian matrix is shown. In this graph the colour scale represents the relative importance of each component. The white points belong to components with the maximum relative importance while the black ones are assigned to components with minimum relative importance.

Several conclusions can be reached from these graphics:

- All the elements belonging to the main diagonal of the matrix are relevant, since every variable has an important influence on their own error functions.

- The variation in any $k$-order harmonic in the AC side exerts a particularly meaningful influence over the DC side harmonic components with orders $k-1$ and $k+1$ and viceversa. This situation leads to the appearance of diagonal structures in certain zones of the matrix. Figure 6 shows a zoom of the graphic presented in figure 5 in the zone corresponding to $\mathrm{AC}-\mathrm{DC}$ interaction. The diagonal lines inside the matrix can be observed in this figure.

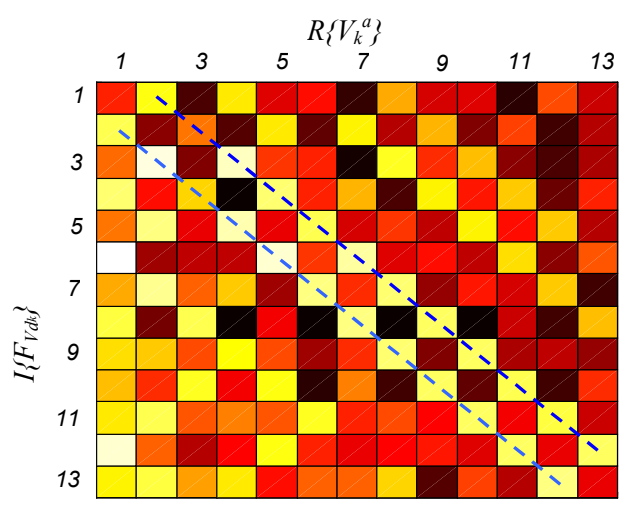

Fig. 6. Zoom of the top view of the Jacobian matrix for the zone corresponding to the AC-DC interaction

- The times of end of conduction $\phi_{i}$, exert a weak influence over the rest of the variables. In fact, their effect is only relevant to their own error functions.

- The times of starting of conduction have a strong influence on the rest of the system variables, especially on their own error functions and on the error functions of end of conduction.

The two last conclusions can easily be understood if it is remembered that the variations in the times of starting of conduction affect both the precursor currents and the convolution pulses. However, the changes in the end of conduction times only affect the convolution pulses. This situation is shown in graph form in figure 7.
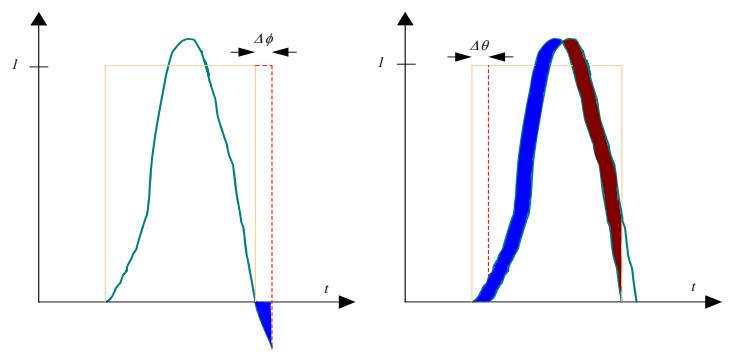

Fig. 7. The Influence of variation in the starting and ending conduction times 


\section{E. Methods for the acceleration of the algorithm}

Several techniques have been proposed for the controlled rectifier operating in the continuous mode to accelerate the execution of the algorithm [2]. The extrapolation of these techniques to the uncontrolled rectifier working in the discontinuous conduction mode is also possible. In fact, two different procedures are suitable for the acceleration of the algorithm for operation in the discontinuous conduction mode.

\section{Single calculation of the Jacobian matrix}

Since the Jacobian matrix is a linearisation of the system around an operation point, it is possible to assume that the values of the partial derivatives will not present drastic changes from one iteration to another. For this reason it is possible to make only the initial calculation of the Jacobian matrix and keep its values constant until the final convergence of the method. If the single calculation of the Jacobian matrix is applied, a larger number of iterations must be made before reaching convergence. However, the saving of time and computation resources is considerable since every iteration of all the elements in a square matrix of order $8 N+13$ need not be calculated.

Although these methods allows the Newton algorithm to converge faster, it should be noted that under certain circumstances the lack of updating in the Jacobian matrix during the iterative process can cause divergence of the method, particularly in those cases where resonance can occur.

\section{Partial updating of the Jacobian matrix}

A less drastic procedure for the acceleration of the algorithm consists of the partial updating of the Jacobian matrix. In this approach, only the elements of the Jacobian matrix that surpass a certain absolute value are updated after the first iteration. Usually the elements to be updated belong to the AC/DC diagonal of the system and are related to the starting and end of conduction times.

A direct application of the method using sequence components, [8], [9] is also possible. This procedure permits the direct calculation of the solution without additional conversions in the case when the desired format of the result are the sequence components, which is a very frequent situation in the analysis of power systems.

\section{Practical Application of the Method}

In this section, a practical case for the application of the method will be presented. The selected case will include a resonance frequency in the analysed spectrum in order to demonstrate the robustness of the method. The next figure shows the system that will be analysed.

The supply system will have a $0.3 \%$ unbalance and the direct sequence component will be delayed $40^{\circ}$ with respect to the inverse sequence component. The complete definition of the supply voltage is presented in table II.

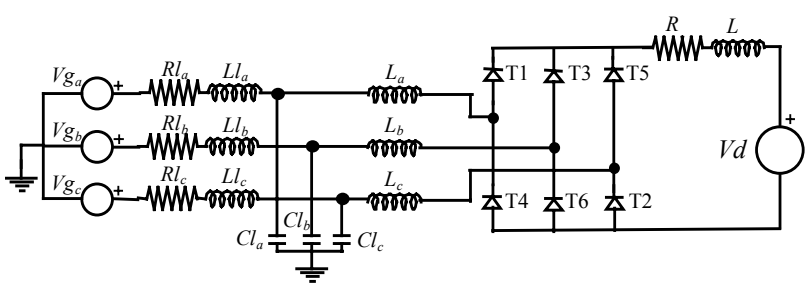

Fig. 8. Test system

TABLE II. - Sequence components of supply voltage

\begin{tabular}{|c|c|c|c|c|c|}
\hline \multicolumn{3}{|c|}{ Positive sequence } & \multicolumn{3}{|c|}{ Negative sequence } \\
\hline$V_{d}$ & $r m s(V)$ & 220 & $V_{i}$ & rms (V) & $220 \cdot 0,003$ \\
\hline & Phase angle ( $\left.{ }^{\circ}\right)$ & 0 & & Phase angle ( $\left.{ }^{\circ}\right)$ & 40 \\
\hline
\end{tabular}

The electrical system consists of a three-phase uncontrolled rectifier supplied through the equivalent impedance of the power network. A capacitor battery for power system compensation has also been included in the connection of the rectifier to the power system. The values of all these components are shown in table III.

TABLE III. - Values of the AC parameters

\begin{tabular}{|c|c|c|c|c|c|}
\hline \multicolumn{3}{|c|}{ Filtering reactances } & \multicolumn{3}{c|}{ Power system impedance } \\
\hline$L_{a}(m H)$ & $L_{b}(m H)$ & $L_{c}(m H)$ & $R l(m \Omega)$ & $L l(\mu H)$ & $C l_{(\mu F)}$ \\
\hline 0.3 & 0.3 & 0.3 & $5 \sqrt{k}$ & 40 & 90 \\
\hline
\end{tabular}

The value given for $R l$ in the above table takes into account the variation caused in the resistance by the "skin" and "proximity" effects. Although a more detailed approach to this phenomenon can be found in [2,pp.41$46]$, in the present simulation the correction factor $5 \sqrt{k}$ has been included in order to show the simplicity of including the dependence of certain system parameters on frequency when the simulation is carried out in the frequency domain. However, in the case of time domain simulation this kind of effect can hardly be modelled.

On the DC side of the system an inductive series impedance connected to a voltage source was included. The source will have a DC level in order to produce discontinuous conduction mode operation in the rectifier. A certain ripple level will also be included in the source as presented in table IV.

TABLE IV. - DC parameters

\begin{tabular}{|c|c|c|c|c|c|c|}
\hline \multicolumn{5}{|c|}{ Thevenin Voltage $\left(V_{T h}\right)$} & \multicolumn{2}{|c|}{ Thev. Imped. $\left(Z_{T h}\right)$} \\
\hline \multicolumn{2}{|c|}{ Harmonics } & \multicolumn{3}{|c|}{$V_{T h 0}=522,72 \mathrm{~V}$} & $R l=0,1 \Omega$ & $L l=0,2 \mathrm{mH}$ \\
\hline \multicolumn{2}{|r|}{ Order } & 1 & 2 & 5 & & \\
\hline \multirow[t]{2}{*}{$V_{T h}$} & Amplitude (V) & 4 & 3 & 2 & & \\
\hline & Phase angle $\left({ }^{\circ}\right)$ & 45 & 60 & 0 & & \\
\hline
\end{tabular}

All the frequencies lower than $3 \mathrm{KHz}$ have been included in the resolution of the system equations. This resolution is equivalent to accounting for the first 60 harmonic components.

For the present system it can be easily demonstrated that a resonance frequency exists at $2650 \mathrm{~Hz}$. Therefore, this example is an excellent test for the convergence properties of the method. 
The example was solved using two different methods. On one hand, the single calculation of the Jacobian matrix was applied in order to accelerate the algorithm convergence. On the other hand, the complete method of total updating of the Jacobian matrix was also used in order to obtain the optimal convergence characteristics and ensure the correct calculation of the solution even under resonance conditions.

The selected convergence criterion consists of limiting the total sum of the absolute error value to a certain value in every error function. The next equation represents the mathematical formulation of this criterion.

$$
\sum_{i=1}^{8 N+13}\left|F_{i}\right|<10^{-6}
$$

In the resolution of this example, by applying the total updating of the Jacobian matrix in every iteration, 8 complete iterations were necessary. However, in the case of a single calculation of the Jacobian matrix 24 iteration were needed. In spite of the higher number of iterations needed in the second case, its computation time was approximately 3 times shorter, which shows the huge saving of mathematical calculation achieved with the use of acceleration methods.

Figure 9 shows a comparison of the evolution of the error vs. number of iterations and computation time for the two different techniques. In figure 10 the final appearance in the time domain of the rectifier supplying currents and the power system currents up from the capacitor bank is presented.

Figure 11 shows the rectifier supplying currents as they are obtained from the direct application of the method in the frequency domain. The positive sequence harmonics have been represented on the positive abscise axis and the negative sequence ones on the negative axis.

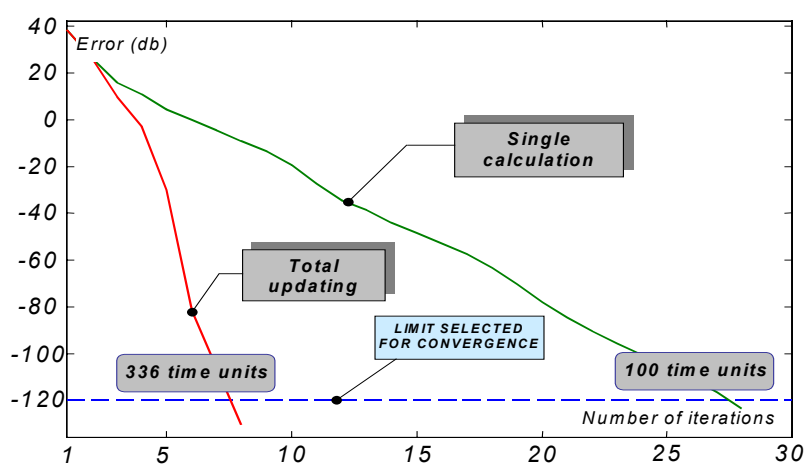

Fig. 9. Error evolution vs. number of iterations and time

The analysed case is useful for the study of the robustness of the algorithm. However, it should be pointed out that the connection of a pure capacitor bank, as indicated in figure 8, would not be a suitable solution for an industrial installation because it could give rise to resonance problems. In fact, antiharmonic inductances are commonly used in industrial plants. These inductances are series connected with the capacitor bank in order to adjust the resonance frequency to values lower than the expected harmonic frequencies (typically under the $5^{\text {th }}$ harmonic). The use of antiharmonic inductances also eliminates the influence on the resonance frequency of the line inductances, which are in this case much lower than the antiharmonic ones.

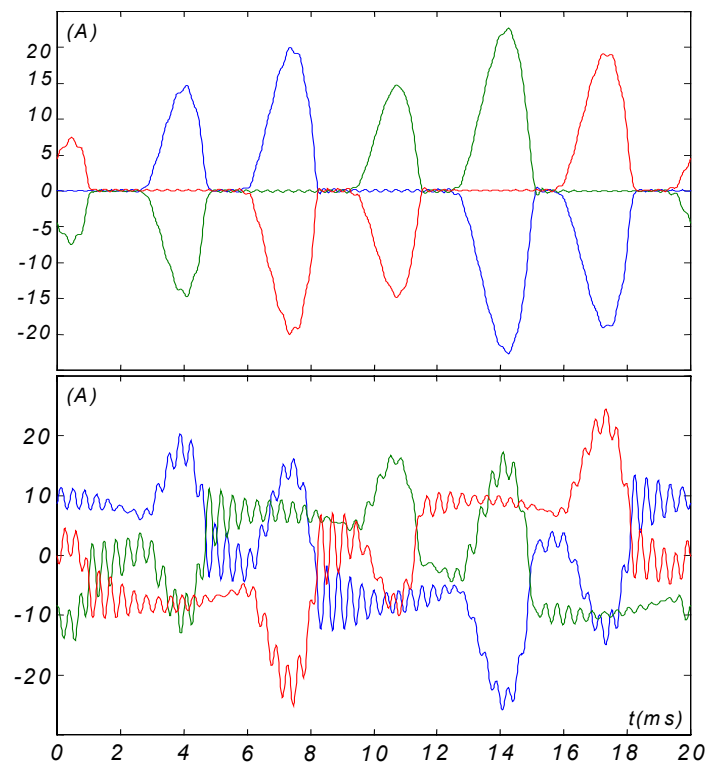

Fig. 10. Rectifier supply currents (top). Power system currents before the capacitor bank (bottom)

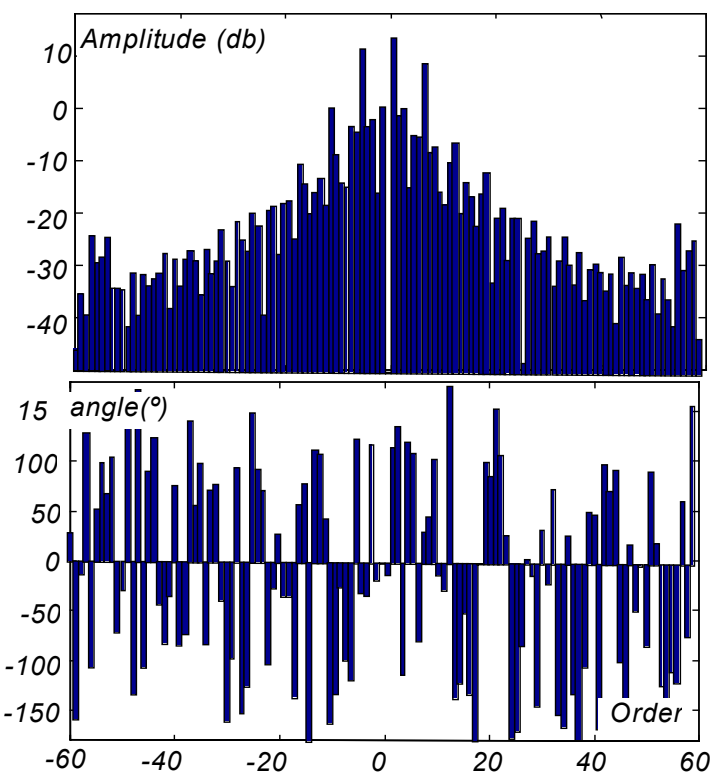

Fig. 11. Rectifier supply currents in the frequency domain

In order to present results that are more realistic, the simulation was repeated after series connecting three antiharmonic inductances to the thee-phase capacitor bank. To calculate the value of the inductances, equation 7 can be applied.

$$
f_{r}=\frac{1}{2 \pi \sqrt{L C}}
$$

A value of $6,7 \mathrm{mH}$ was selected in order to tune the resonance frequency below the $5^{\text {th }}$ harmonic. The results calculated for the new system are presented in figure 12 . In this figure the effect caused by the antiharmonic 
inductances can be observed (elimination of the ripple present in the power system currents). This ripple was caused by the proximity between the resonance frequency and some relevant harmonic components in the line current.
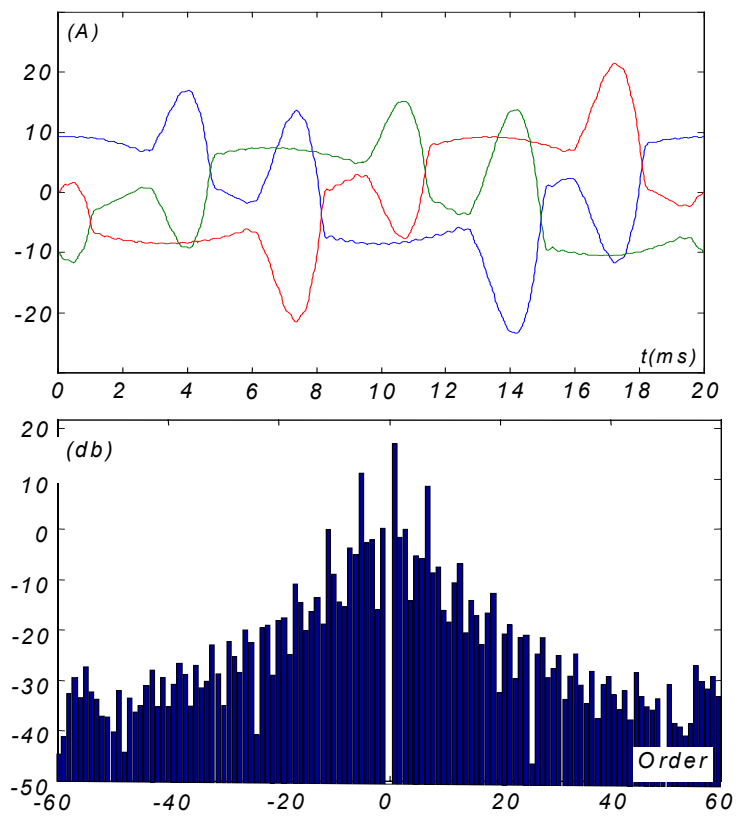

Fig. 12. Power system currents after the installation of antiharmonic inductances

\section{Conclusions}

A new model for the calculation of the AC/DC interaction in 6 pulse uncontrolled rectifiers has been presented. This new model, by means of an iterative algorithm, allows the analysis and quantification of the effects caused to the system by this non-linear loads, taking into account even the influence of frequency in systems parameters.

The Gauss method has proved itself to be the simplest; however, its convergence is much slower and the mathematical resolution of the problem is not possible if the starting point for iteration is not close to the real solution. This situation strongly reduces the scope of application of the method, especially in the calculation of DC variables where the presence of a moderate level of voltage ripple can lead to the divergence of the algorithm if an ideal DC voltage is taken for the first iteration.

The Newton method provides a series of important improvements on the Gauss method. Convergence is faster and the probability of divergence of the algorithm is much more limited. The main drawback of this method is the need for a very precise knowledge of the system in order to obtain an accurate calculation of the Jacobian matrix.

The numerical calculation of all partial derivatives needed to obtain the Jacobian matrix has shown itself to be a process that involves a huge consumption of time and computational resources. A possible solution to this problem is the analytical calculation of the mathematical relations between variables. This kind of calculation involves a big initial effort, but once the analytical expressions have been obtained the reduction in computation time is really remarkable.

Although the usual acceleration techniques for the Newton's algorithm have proved their usefulness in the reduction of computation time and resources, its effect on convergence must be also taken into account, especially in those cases where the possibility of resonance phenomena exist.

\section{Acknowledgement}

The authors would like to gratefully acknowledge the financial support given by the Education and Culture Department of the Regional Government of Asturias through funds from the Research, Technological Development and Innovation Plan $(\mathrm{I}+\mathrm{D}+\mathrm{I})$ of Asturias 2000-2004.

\section{References}

[1] J.M. Cano. (2000) "Funciones de Transferencia para el análisis en distintas condiciones de funcionamiento de la distorsión armónica asociada a rectificadores trifásicos ", Tesis doctoral, Universidad de Oviedo.

[2] J. Arrillaga, B.C. Smith, N.R. Watson, A.R.. Wood. (1997) "Power System Harmonic Analysis", John Wiley \& Sons.

[3] C. Callaghan, J. Arrillaga. (1994) " A double iterative algorithm for the analysis of power and harmonic flows at ac-dc terminals ", Proc. IEE, 136(6), 319-324.

[4] E.V. Larsen, D.H. Baker, J.C. McIver. (1989) " Low-order Harmonic Interactions on AC/DC Systems ", Transactions on Power Delivery, Vol. 4, $\mathrm{N}^{\mathrm{o}} 1$, pp. 493-501.

[5] B.C. Smith. (1996) "A harmonic domain model for the interaction of the HVdc convertor with ac and dc", Tesis doctoral, Universidad de Canterbury, Nueva Zelanda.

[6] B.C. Smith, N.R. Watson, A.R. Wood, J. Arrillaga. (1996) "Newton solution for the steady-state interaction of AC/DC systems", IEE Proc.-Gener. Transm. Distrib., Vol. 143, No 2, pp. 200-210.

[7] J.M.Cano, G.A.Orcajo, J.G.Mayordomo, R.Asensi, M.F.Cabanas, M.G.Melero. (2000) "Distortion Analysis in $\mathrm{AC} / \mathrm{DC}$ rectifiers operating in discontinuous mode under unbalanced conditions", Proc. Power Quality Conference PQC, pp. 137-131.

[8] B.C. Smith, N.R. Watson, A.R. Wood, J. Arrillaga. (1997) "A Sequence components model of the ac/dc converter in the harmonic domain", IEEE

Transactions on Power Delivery, Vol. 12, No 4, pp. 1736-1743.

[9] B.C. Smith, J. Arrillaga. (1999) "Power Flow Constrained Harmonic Analysis in AC-DC Power Systems", IEEE Transactions on Power Systems, Vol. 14, No 4, pp. 1251-1261. 